\title{
Germanica
}

\section{Joseph Roth, Stefan Zweig et Soma Morgenstern : correspondance et souvenirs}

Joseph Roth, Stefan Zweig und Soma Morgenstern: Korrespondenz und Erinnerungen

\section{Erika Tunner}

\section{(2)enEdition}

\section{Journals}

Édition électronique

URL : http://journals.openedition.org/germanica/569

DOI : $10.4000 /$ germanica.569

ISSN : 2107-0784

\section{Éditeur}

Université de Lille

\section{Édition imprimée}

Date de publication : 1 décembre 2008

Pagination : 123-130

ISBN : 978-2-913857-22-6

ISSN : 0984-2632

Référence électronique

Erika Tunner, « Joseph Roth, Stefan Zweig et Soma Morgenstern : correspondance et souvenirs », Germanica [En ligne], 43 | 2008, mis en ligne le 01 décembre 2010, consulté le 06 octobre 2020. URL http://journals.openedition.org/germanica/569; DOI : https://doi.org/10.4000/germanica.569

Ce document a été généré automatiquement le 6 octobre 2020 .

(c) Tous droits réservés 


\title{
Joseph Roth, Stefan Zweig et Soma Morgenstern : correspondance et souvenirs
}

\author{
Joseph Roth, Stefan Zweig und Soma Morgenstern: Korrespondenz und
}

Erinnerungen

Erika Tunner

1 Trois écrivains dont Joseph Roth, né en 1894 est le plus jeune. Soma Morgenstern, son aîné de 4 ans, issu d'une famille juive hassidique est, comme Roth, originaire de la Galicie orientale, à l'époque une province de l'empire d'Autriche. Ils se rencontrent pour la première fois en 1910 à Lemberg, lors d'une conférence des lycéens sionistes de Galicie. Puis, étudiants, les deux jeunes gens commencent à se fréquenter dès 1913 à Vienne, la ville natale de Stefan Zweig dont Roth a fait la connaissance en 1929 seulement quand Zweig alors âgé de 48 ans, était déjà parvenu au faîte de la gloire littéraire. Trois écrivains qui, au cours de leur vie, ont fait l'expérience d'une société plurielle contrastée qui favorise les échanges et leur inspire par ailleurs des identifications multiples. Enfin, trois écrivains juifs "en errance", Juden auf Wanderschaft, citoyen du monde, comme Stefan Zweig, citoyens des hôtels comme Joseph Roth et Soma Morgenstern, cosmopolites par conviction, apatrides par nécessité. Soma Morgenstern et Stefan Zweig ont été liés à Joseph Roth par une amitié solide et ont accompagné une grande partie de son œuvre. Mort à New York en 1976, Morgenstern reste le moins connu des trois et ce n'est qu'assez tardivement que son œuvre a été traduite en français. Journaliste de profession, il possède pourtant aussi un beau talent de conteur et sait analyser avec finesse les hommes et les événements.

La Galicie, avec son mélange ethnique de Juifs, de Polonais, de Ruthènes et d'Allemands signifiait pour Roth la richesse du brassage des peuples. D'autres écrivains originaires de cette région, comme Karl Emil Franzos, Leopold von Sacher-Masoch, puis Manès Sperber, ont marqué fortement la littérature de langue allemande ${ }^{1}$. À Brody, la ville natale de Roth, on parlait aussi bien allemand, ukrainien, polonais que yiddish; Roth apprenait l'hébreu et savait assez de russe pour déchiffrer l'écriture cyrillique des 
journaux russes. Dès 1920, il se met à étudier le français et se passionne pour Flaubert et Stendhal. Autant d'atouts pour comprendre l'originalité des différentes mentalités et de transférer leur traits significatifs à de nombreux personnages dans son œuvre littéraire. Toutefois, dans le monde occidental, et notamment à Berlin où Roth s'était rendu en 1920, la Galicie n'avait pas bonne presse et venir de ce pays suscitait plus de répulsion que d'attirance: "Les Berlinois parlaient de la Galicie avec une haine sans commune mesure avec celle qu'ils pouvaient avoir pour tout autre pays. Aucun homme ne se formalisait qu'il fût question de la Roumanie, de la Bulgarie, de la Serbie ou du Monténégro. Mais dès que tombait le mot 'Galicie', tout le monde faisait le dégoûté ", écrit un des compatriotes juifs de Roth, l'acteur Alexander Granach, dans le récit de sa vie intitulé Da geht ein Mensch (Là, marche un homme) ${ }^{2}$. À la fin des années trente, Roth lui-même revient sur ce problème dans un entretien avec sa compagne d'alors, la romancière Irmgard Keun : «Plus le lieux d'origine du juif est occidental, plus il a des juifs qu'il regarde de haut. Le juif de Francfort méprise le juif de Berlin, le juif de Berlin méprise le juif de Vienne, le juif de Vienne méprise le juif de Varsovie. Puis, bien audelà, viennent encore les juifs de Galicie que tous les autres regardent de haut, et c'est de là que je viens, moi, le dernier des derniers des juifs $»^{3}$.

Rien de tel pour Stefan Zweig, fils d'une riche famille de la grande bourgeoisie, né à Vienne et marqué par la culture de la ville impériale où il était tentant de croire en une communauté spirituelle des élites par-dessus les frontières. Cependant, lui aussi, dans un de ses récits les plus émouvants, Buchmendel (Le Bouquiniste Mendel), place au centre de l'histoire un Juif de Galicie, occupé à ses livres, qui se heurte aux autorités policières et finit par être envoyé dans un camp d'où il ressortira deux ans plus tard, définitivement brisé...

Paradoxalement, Zweig évite de se fixer à Vienne : certes, dans le "monde d'hier", dans cet " âge d'or de la sécurité " avant la Première Guerre mondiale, «chaque chose avait sa norme, sa mesure et son poids déterminé $»^{4}$. On pouvait y mener une vie facile, insouciante, surtout lorsqu'on disposait d'une certaine fortune. Mais Zweig ne s'y sent pas vraiment chez lui. Dès 1905, il s'en plaint périodiquement à ses amis ou bien confie son malaise à son journal intime. En partie pour y échapper, il fait des voyages avec une fréquence étonnante. Dans ses essais, il cherche un dialogue avec les plus grands et les plus divers esprits du passé, interrogeant tour à tour Stendhal et Balzac, Tolstoï et Dostoïevski, Freud et Mesmer, Dickens, Casanova, Hölderlin, Nietzsche, pour ne citer que quelques-uns. Dès 1902, il se fait l'inlassable avocat de la littérature française, grâce à ses nombreuses traductions. Toute sa vie, il va correspondre avec des intellectuels français et œuvrer pour le rapprochement des cultures. À la fin de l'année 1914, il propose à son ami Romain Rolland de fonder dès la fin de la guerre une revue européenne réunissant des auteurs français et allemands : elle vit le jour en 1920, sous le titre Europe. D'une qualité remarquable, elle existe toujours et fait découvrir et redécouvrir à un large public les grands écrivains du monde entier.

Roth, lui, s'était tourné très tôt vers Heine avec lequel il se sentait d'étroites affinités et dont l'œuvre a été souvent présente à son esprit pendant ses séjours à Berlin, Francfort et Paris. Comme Heine, Roth était un écorché vif, comme Heine, il était d'un tempérament à la fois exalté et rationnel, comme Heine encore, il savait protéger sa sensibilité excessive par le sens de l'ironie voire par un certain cynisme. L'un et l'autre ont longtemps gagné leur vie comme journalistes et ils ne vomissaient pas le journalisme comme un Karl Kraus ou un Fritz Mauthner, plaçant cependant toujours 
leur véritable vocation dans la création littéraire. Pour l'un et l'autre, Paris a d'abord été un refuge, et c'est finalement à Paris que s'est terminé le parcours de leur existence. D'après les propos rapportés par Morgenstern, c'est aussi à Paris que Roth semble avoir pris conscience de la véritable orientation de son écriture : « ... en lisant Proust, s'est produit le déclic : j'ai su comment je devais écrire ${ }^{5}$.

Bien que Vienne représente pour Roth le contact avec la manière de penser occidentale et exerce sur lui une forte attraction, il éprouve, lui aussi, un malaise certain dans cette ville où il assiste, dès 1913, aux frictions entre les différentes nationalités, aux poussées de fièvres nationalistes, aux manifestations de sentiments antisémites. A Vienne, à l'époque le lieu d'attache de la plus grande communauté juive d'Europe centrale, il est pourtant «terriblement dur d'être un juif de l'Est », estimait Roth, « il n'y a pas de sort plus dur à Vienne que celui du juif de l'Est étranger » avoue-t-il dans son ouvrage Juifs en errance dans lequel il décrit indirectement son propre destin ${ }^{6}$. Roth et Morgenstern assistent tous deux aux affrontements entre les nationaux-allemands antisémites, précurseurs des nazis, et les étudiants juifs, soutenus par les étudiants yougoslaves et tchèques ${ }^{7}$. Arthur Schnitzler en parle longuement dans son autobiographie Jugend in Wien (Une jeunesse viennoise) où il évoque aussi le fameux décret de Waidhofen, de 1896, dont il n'est peut-être pas inutile de rappeler un bref extrait :

Tout fils d'une mère juive, tout homme dans les veines duquel coule du sang juif, est, de par sa naissance sans honneur, et dénué de tout sentiment délicat. Il ne peut faire la différence entre ce qui est sale et ce qui est pur. C'est un sujet moralement inférieur. C'est pourquoi il est déshonorant d'entretenir des relations avec un juif8.

7 Ce n'est qu'à Paris où Roth arrive en 1925 qu'il perd un peu le sentiment de sa marginalité. «Les juifs de l'Est ont même des raisons extérieures qui leur rendent la vie facile à Paris », écrit-il dans Juifs en errance, « leur physionomie ne les trahit pas. Leur vivacité n'attire pas l'attention. Leur sens de l'humour est à mi-chemin de l'esprit français. Paris est une véritable métropole. Vienne en a été une autrefois. Berlin en sera une autre plus tard $»^{9}$. Il est vrai cependant que même à Paris il n'a pas toujours échappé à des propos antisémites qui le blessaient toujours profondément.

Ses pérégrinations journalistiques mènent Roth à plusieurs reprises à Prague, cette métropole multiculturelle où cohabitent les populations tchèque, juive et germanophone et où il publie des textes dans le Prager Tagblatt. Roth conservait des relations avec ce journal jusqu'à la fin des années vingt. A son rédacteur en chef, il recommande un jeune ami français, Pierre Bertaux, étudiant puis lecteur à Berlin et plus tard germaniste de renom, avec lequel il entretient des relations particulièrement cordiales : «Monsieur Pierre Bertaux est l'un des Français les plus aimables et mon ami ", écrit Roth, «il est studieux, bien élevé et suffisamment ironique pour comprendre le ton de notre patrie intellectuelle, Prague $»^{10}$. Parmi les intellectuels de Prague, mosaïque de cultures marquées par des conflits et des contradictions, Roth fréquente surtout Johannes Urzidil et s'entretient avec lui de la monarchie des Habsbourg et du monde slave de l'Est, deux sujets qui restent, presque obsessionnellement, au centre de ses préoccupations. Quant à Urzidil, il donne dans ses romans les images à la fois les plus cauchemardesques et les plus idylliques de Prague, d'abord dans la nouvelle Das Gespenst in der Judenstadt (Le spectre de la ville juive), et, bien plus tard, dans le Prager Triptychon (Le Triptyque de Prague) ${ }^{11}$.

9 Les discussions sur le judaïsme et sur l'antisémitisme, mais aussi les analyses politiques, les échanges sur la littérature et même sur les problèmes personnels marquent les 
relations de Joseph Roth avec Soma Morgenstern, le plus fidèle de ses amis qui l'a accompagné dans sa jeunesse à Vienne, qui a travaillé avec lui dans les années vingt comme correspondant pour la Frankfurter Zeitung, à l'époque le journal le plus un vue de l'Allemagne. Fuyant le nazisme, il se réfugie en 1938 à Paris où il loge dans le même hôtel que Roth et ne le quitte pas jusqu'à sa mort en 1939.

Morgenstern retrace son amitié avec Joseph Roth dans son captivant livre de souvenirs, Joseph Roths Flucht und Ende (Fuite et fin de Joseph Roth), allusion au roman de Roth paru en 1927, Flucht ohne Ende (Fuite sans fin). Dans la fuite, Morgenstern reconnaitt un motif essentiel de la vie de son ami. "Il fut dès le début un fugitif volontaire ", écrit-il et

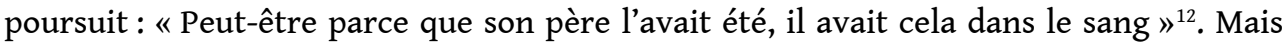
c'est peut-être aussi, plus généralement, un signe distinctif des Juifs de l'Est. Roth luimême le suggère dans Juifs en errance : «Beaucoup partent (...) Les juifs de l'Est n'ont de patrie nulle part mais ils ont des tombes dans tous les cimetières $»^{13}$. Les nombreux voyages de Roth peuvent être compris, eux aussi, comme un des modes de la fuite. Avec cette clairvoyance qui est celle de l'amitié, Morgenstern note dans son journal : «Ce qui attirait Roth dans les voyages, c'était d'être ailleurs, voire nulle part, plutôt que chez soi. (...) Pourtant, il souffrait toujours, comme d'un mal aigu, d'être sans patrie (...). Voyager était sa seconde façon de se griser. C'est ainsi que la fuite devint sa patrie ${ }^{14}$. La première façon de se griser, l'absorption constante d'alcool, autre véhicule de la fuite, devait devenir un des fils conducteur de l'ouvrage de Morgenstern et un de ses soucis majeurs au cours de leur amitié.

11 Il faut dire que Morgenstern semble avoir eu un don particulier pour l'amitié dont il avait du reste parfaitement conscience lui-même : "Je peux dire sans exagération que ce fut la bénédiction de ma vie ", écrit-il dans son ouvrage consacré à son idole Alban Berg, publié à titre posthume seulement ${ }^{15}$. Musil, à son tour proche de Morgenstern, tenait son œuvre littéraire en grande estime et le félicitait chaleureusement à propos de son roman Der Sohn des verlorenen Sohnes (Le fils du fils prodigue) dont il affirmait qu'il appartient décidément à la littérature mondiale. En dépit de cet éloge exceptionnel, les écrits de Morgenstern ont longtemps sombré dans l'oubli dont ils émergent peu à peu depuis la fin du siècle dernier.

12 Si ce n'était pas dans la cour de l'université de Vienne, c'était au Café Museum que Roth et Morgenstern se donnaient rendez-vous, c'était aussi au Café Museum que Roth, grâce à Morgenstern, rencontra Musil qui manifesta à Roth toute l'admiration que lui avait inspirée son roman Hiob. Roman eines einfachen Mannes (Job. Roman d'un homme simple, plus connu en France sous le titre Le poids de la grâce). "Le café à Vienne n'est pas seulement un café, c'est une conception du monde ", remarqua un jour Alfred Polgar ${ }^{16}$. En effet, le 'Wiener Kaffeehaus', lieu de rencontres et d'échanges, de refuge et de retraite, est une spécificité culturelle viennoise et un véritable phénomène social qui fait partie d'un mode de vie. Il devient le lieu de la modernité comme le montre Béatrice Gonzalès-Vangell dont la très belle thèse Kaddish et Rennaissance. La Shoah dans les romans viennois (1991-2001) comporte un important chapitre sur l'histoire et la vocation du Kaffeehaus. Dans Le monde d'hier, Stefan Zweig se remémore le temps de ses études et affirme : « la meilleure de toutes les écoles pour tout ce qui était neuf, restait le Kaffehaus $»^{17}$. Avec le Café Museum, le Café Herrenhof a été l'établissement préféré de Joseph Roth ; en 1919 il y fait la connaissance d'une ravissante jeune fille, Friederike Reichler, «Friedl », qu'il épouse trois ans plus tard. Mais Roth n'était pas seulement un habitué des 'Wiener Kaffeehäuser'. Pour lui, qui n'a jamais connu un «chez soi », les 
cafés de Berlin, Francfort, Amsterdam, Bruxelles, Paris, Nice ou Marseille représentent dans sa vie de nomade des endroits d'un espace convivial et protégé qui lui permettent de bavarder mais aussi de s'isoler intérieurement pour travailler. Pendant les années d'émigration à Paris (1933-1939), c'est près du Sénat, dans la rue de Tournon, d'abord au café de l'Hôtel Foyot, puis, après la destruction de celui-ci, dans le café de la Poste, situé dans la même rue, que ses amis, des réfugiés juifs comme des catholiques et des monarchistes, se retrouvaient autour de Roth, plongé dans ses écrits mais en même temps toujours extrêmement attentif aux autres et prêt à rendre service. Son opposition au nazisme a été virulente et se manifestait en partie par le souhait de restituer la monarchie habsbourgeoise pour faire face à l'Allemagne hitlérienne. De l'argent, Roth n'en avait que par intermittence, mais sa prodigalité était légendaire comme c'est souvent le cas chez ceux qui ont le sens aigu de la précarité des choses et de la marche inexorable du temps. Morgenstern rapporte qu'il n'a jamais entendu un homme parler autant d'argent que Roth dans les derniers temps de leur vie commune à Paris : il avait besoin d'argent, il attendait un chèque, un chèque était en retard, un mandat n'était pas arrivé, un éditeur l'escroquait. Et pourtant, poursuit Morgenstern, il est probablement peu d'écrivains et vraisemblablement très peu de gens qui avaient autant méprisé l'argent ${ }^{18}$.

13 C'est avec Zweig que Roth mène le plus long dialogue épistolaire pendant une bonne dizaine d'années. Les lettres des six dernières années de sa vie, qu'il passe à Paris tandis que Zweig se trouve à Londres, représentent un précieux témoignage permettant de saisir sur le vif les problèmes discutés dans les milieux de l'émigration littéraire allemande et autrichienne ${ }^{19}$. On peut dégager plusieurs lignes de force qui constituent la trame de cette correspondance : l'amour de la lecture et de l'écriture ; l'attachement à la langue allemande; la fascination éprouvée pour la France; la justesse parfois visionnaire des analyses politiques; l'affirmation intransigeante de la responsabilité morale de l'artiste et, plus marginalement, la « question juive ». En fait, Zweig élude sa définition en tant que Juif, notamment face à l'antisémitisme, tandis que chez Roth, vers la fin de sa vie, le catholicisme semble se superposer à son judaïsme initial, selon la thèse, brillante au demeurant, de Jacqueline Bel, intitulée Péché et rédemption ou de l'apaisement du mal d'être : une approche nouvelle de l'œuvre et de l'écrivain Joseph Roth, 1992, une analyse de l'évolution du sentiment religieux chez Roth, centrée sur certains textes, en particulier son dernier récit, Die Legende vom heiligen Trinker (La légende du saint buveur). En dépit de ce travail fort bien argumenté, les avis restent partagés et Roth lui-même n'a pas été homme à prendre position d'une manière parfaitement univoque.

14 Zweig, l'ami «paternel » de Roth, prend la place de ce père que Roth n'a jamais connu et qui lui a cruellement manqué. En dépit de quelques refroidissements passagers, leur attachement réciproque ne s'est jamais vraiment relâché. Avec beaucoup de générosité Zweig a toujours accordé à Roth un soutien autant matériel que moral. Il le supplie sans cesse de se ménager, de réduire sa consommation d'alcool. Mais Roth lui dit sans ambages qu'il ne peut écrire que lorsqu'il a bu, et qu'il est perdu s'il ne peut pas écrire "J'écris chaque jour uniquement pour me perdre dans des destins fictifs », "Je travaille pour fuir $\aleph^{20}$. À la même époque, il affirme dans en entretien avec le critique Frédéric Lefèvre, publié dans les Nouvelles Littéraires le 2 juin 1934 : «Je travaille dix heures par jour (...) Je suis un ouvrier consciencieux de la langue. La langue allemande est ma 
patrie, la langue française une amie que j'aime de tout cœur, et qui me donne l'hospitalité. [...] » $»^{21}$.

Deux romans ont consacré la réputation de Joseph Roth : Hiob. Der Roman eines einfachen Mannes, 1930 (traduit en français dès 1931 sous le titre Job, roman d'un simple juif) et Der Radetzkymarsch, 1932, (La Marche de Radetzky, traduit en 1934): ils représentent l'évocation littéraire des deux univers qui ont été les siens, et qui, dépassant le cadres de ses préoccupations personnelles, sont particulièrement représentatifs du genius loci des villes de la Mitteleuropa : le monde du judaïsme et l'Autriche-Hongrie de François Joseph. Joseph Roth, Soma Morgenstern, Stefan Zweig : trois voix singulières au carrefour des échanges et des transferts possibles mais aussi témoins lucides des tribulations de leur temps.

\section{NOTES}

1. Cf. à ce sujet : Galizien, eine literarische Heimat, hg. Von Stefan H. Kaszynski, UaM, Poznan, 1987.

2. In : David Bronsen : Joseph Roth (traduction de René Wintzen), Paris, Seuil, 1994, p. 20.

3. Ibid., p. 27.

4. Stefan Zweig: Le Monde d'hier (traduction de Jean-Paul Zimmermann), Paris, Belfond, 1982, pp. 17-18.

5. Propos rapportés par Soma Morgenstern in : Fuite et fin de Joseph Roth (traduction de Denis Authier), Paris, Liana Levi, 1997, p. 116.

6. In : David Bronsen, op. cit., p. 74.

7. Soma Morgenstern, op. cit., p. 10.

8. Arthur Schnitzler, Une jeunesse viennoise (traduction de Nicole et Henri Roche), Paris, Hachette, 1987, p. 198 et aussi p. 408.

9. In : David Bronsen, op. cit., p. 136.

10. Texte d'une carte postale, propriété privée, in : David Bronsen, op. cit., p. 118.

11. Cf. à ce sujet Erika Tunner : «' Wir in Prag' . L'image de Prague dans la mémoire de Johannes Urzidil », in : Allemands, Juifs et Tchèques à Prague/Deutsche, Juden und Tschechen in Prag, 1890-1924, sous la direction de Maurice Godé, Jacques Le Rider et Françoise Mayer, Bibliothèque d'Etudes Germaniques et Centre-Européennes, Université Paul-Valéry de Montpellier, 1996, p. 451-461.

12. Postface à son livre sur Roth, op. cit, p. 355.

13. In : David Bronsen, op. cit., p. 144.

14. Postface à son livre sur Roth, op. cit, p. 356.

15. «Was Freundschaft betrifft, habe ich in meinem Leben ein besonderes Glück gehabt. Ich kann ohne Übertreibung sagen, dass es der Segen meines Lebens war", in : Alban Berg und seine Idole (Alban Berg et ses idoles), Dietrich zu Klampen Lüneburg, 1995, und Aufbau Vlg., Berlin, 1999, p. 122.

16. In : David Bronsen, op.cit., p. 101.

17. Béatrice Gonzalès-Vangell, Kaddish et Renaissance. La Shoah dans les romans viennois (1991-2001) de R. Schindel, R. Menasse et D. Rabinovici, Villeneuve d'Ascq, Presses Universitaires du Septentrion, 2005, p. 169. 
18. Soma Morgenstern, op. cit., p. $122 \mathrm{~s}$.

Sur Morgenstern, cf. aussi Erika Tunner: “Konstruktionen von Identitäten: der Fall Morgenstern", in : Ambivalenz des kulturellen Erbes. Vielfachcodierung des historischen Gedächtnisses, Paradigma Österreich, hg. von Moritz Csaky und Klaus Zeyringer, Innsbruck, Studienverlag, 2000, p. 179-189.

19. Elles sont disponibles en français dans la traduction de Stéphane Pesnel in : Joseph Roth. Lettres choisies (1911-1939), Paris, Seuil, 2007, annotées et assorties d'une préface remarquable.

20. David Bronsen, op.cit., p. 283.

21. In : Heinz Lunzer, in Zusammenarbeit mit Viktoria Lunzer-Talos, Joseph Roth im Exil in Paris 1933 bis 1939, Wien, Zirkular, 2008, p. 79.

\section{RÉSUMÉS}

Un regard sur ces trois auteurs permet de mettre en lumière quelques aspects fondamentaux des relations et des échanges qui s'établissent entre Vienne, Berlin, Prague et Paris, et des liens entretenus entre trois écrivains juifs, marqués par les lieux de leur origine (la Galicie, la ville de Vienne) autant que par les tribulations de l'Histoire, la montée du nazisme, la confrontation à un antisémitisme virulent, les problèmes aigus de l'émigration, mais aussi par l'expérience de l'amitié et du souci constant de la création littéraire.

Ein Blick auf diese drei Autoren erlaubt es, einige grundlegende Aspekte der Verbindungen zwischen den Metropolen Wien, Berlin, Prag und Paris herauszustellen. Untersucht werden die Beziehungen dreier jüdischer Schriftsteller, die in besonderer Weise geprägt sind von ihrer Herkunft (Galizien, Wien) sowie von den Wirrnissen der Geschichte, dem aufkommenden Nationalsozialismus, der Konfrontation mit einem virulenten Antisemitismus, den schwerwiegenden Problemen der Emigration, aber auch von der Erfahrung der Freundschaft und dem beständigen Gedanken an ihr künstlerisches Schaffen.

\section{INDEX}

Mots-clés : correspondance, souvenir

\section{AUTEURS}

\section{ERIKA TUNNER}

Université Paris XII 